\title{
URBAN AREA EXTRACTION USING AIRBORNE X-BAND FULLY POLARIMETRIC PI-SAR2 IMAGERY
}

\author{
J. Susaki*, M. Kishimoto \\ Department of Civil and Earth Resources Engineering, Graduate School of Engineering, Kyoto University; \\ Email: \{susaki.junichi.3r, kishimoto.masaaki.88n\}@kyoto-u.ac.jp
}

\author{
Commission I, III, VII WG I/2, ICWG III/VII
}

KEY WORDS: PiSAR-2, Urban area, VHR SAR, Fully polarimetric SAR

\begin{abstract}
:
In this paper, we present a method to extract urban areas from X-band fully polarimetric synthetic aperture radar (SAR) data. It is known that very high resolution (VHR) SAR can extract buildings, but it requires more processes to map urban areas that should include other objects. The proposed method is mainly composed of two classifications. One classification uses total power of scattering and volume scattering derived by using four component decomposition method with correction of the polarization orientation angle (POA) effect. The other classification uses polarimetric coherency between $S_{H H}$ and $S_{V V}$. The two results are intersected and final urban areas are extracted after post-classification processing. We applied the proposed method to airborne X-band fully polarimetric SAR data of Polarimetric and Interferometric Airborne Synthetic Aperture Radar System (Pi-SAR2), developed by the National Institute of Information and Communications Technology (NICT), Japan. The validation show that the results of the proposed method were acceptable, with an overall accuracy of approximately 80 to $90 \%$ at $100-\mathrm{m}$ spatial scale.
\end{abstract}

\section{INTRODUCTION}

Human settlement extent data play an important role in discussing urban development and natural resources preservation. When urban areas are rapidly growing, that is the case especially in the developing countries, environmental issues will be caused consequently, e.g. traffic congestion, degradation of water quality and traffic conjestion. The data about urban areas are useful to understand the current situation and tackle with these issues. Synthetic aperture radar (SAR) has potential to monitor urban areas because man-made structures return relatively strong backscattering to distinguish from other objects. Therefore, SAR images have been applied to detect the buildings damaged by disasters (Cossu et al., 2012). Gamba et al. (2011) reported a method to map human settlement extents using VHR SAR in Asia, Europe and Africa. The method relied on the use of local indicators of spatial association (L.I.S.A.) with combination of texture measure, Gray-Level Co-occurrence Matrix (GLCM) textures. The validation results in several study areas show the method did not generate high overall accuracies. Because SAR images may contain the backscattering of objects structure, statistical texture measures may have difficulty in detecting such objects and thus mapping human settlement extents.

In addition to traditional SAR, very high resolution (VHR) SAR has been examined to extract distribution of buildings in urban areas. For example, the mechanism of backscattering from buildings are modeled and simulated (Brunner et al., 2010; Brunner et al., 2011). With the similar approaches, buildings are extracted (Ferro et al., 2013), building heights are retrieved (Brunner et al., 2010) and building change is detected (Marin et al., 2014). However, actual backscattering mechanism is complicated in urban areas. Ferro et al. (2011) studied the relationship between the double-bounce effect of buildings and the orientation angles in VHR SAR images. Kajimoto and Susaki (2013b) also reported the experimental results in an anechoic room and the total powers of backscattering and the four components (Yamaguchi et al.,

\footnotetext{
${ }^{*}$ Corresponding author.
}

2005) derived from fully polarimetric scattering are deeply dependent on the orientation angles. This issue is quite critical in extracting urban areas and buildings in urban areas.

Airborne VHR SAR has been operated in Japan over the last decades. For example, the National Institute of Information and Communications Technology (NICT) and Japan Aerospace Exploration Agency (JAXA), Japan jointly developed fully polarimetric Polarimetric and Interferometric Synthetic Aperture Radar (Pi-SAR). The systems are composed of separate X-band $(1.5 \mathrm{~m}$ slant range resolution) and L-band (3.0 m slant range resolution) radar systems, respectively. After these sensors, NICT developed $\mathrm{X}$-band fully polarimetric Polarimetric and Interferometric Airborne Synthetic Aperture Radar System (Pi-SAR2). The slant range resolution has been improved to $0.3 \mathrm{~m}$. JAXA also developed another L-band radar system, Pi-SAR2-L2 with $1.7 \mathrm{~m}$ slant range resolution. Both systems have been used to monitor the damage caused by disasters, e.g. earthquakes (Satake et al., 2012) and volcanic eruption (Kobayashi et al., 2013). However, the performance to monitor urban areas has not been well examined.

In previous researches, we have already reported a method to extract urban areas by using Advanced Land Observing Satellite (ALOS) / Phased Array type L-band Synthetic Aperture Rader (PALSAR) imagery (Kajimoto and Susaki, 2013a) and another method to estimate urban densities by using a single fully porlarimetric image (Kajimoto and Susaki, 2013b; Susaki et al., 2014). These supervised methods assume to use L-band fully polarimetric SAR (PolSAR) images, but it is not guaranteed that they perform against X-band PolSAR images. Therefore, in this research, we examined the possibility to apply Pi-SAR2 imagery to extracting urban areas.

The remainder of this paper is organized as follows. The location and data collected from the site are described in Section 2. An explanation of the proposed method is given and the experimental results are reported in Section 3. The implications of these results and the validity of the algorithm are then discussed in Section 4. 
Finally, Section 5 concludes the paper.

\section{DATA AND STUDY AREAS}

As mentioned in "1. Introduction", airborne Pi-SAR2 is now operated by NICT, Japan. It measures X-band fully polarimetric scattering. The specification of Pi-SAR2 is listed in Table 1 (Uemoto et al., 2011).

The coverage measured by Pi-SAR2 data is limited. We selected two cities as study areas, Kobe (study area 1) and Kyoto (study areas 2 and 3), Japan among the areas observed because they have several categories of land covers, e.g. urban, mountain and waterbody. We selected two areas among Kyoto: central Kyoto (study area 2) and western Kyoto (study area 3). Figure 1 shows the Advanced Land Observing Satellite (ALOS)/Advanced Visible and Near Infrared Radiometer type 2 (AVNIR-2) optical images of the three study areas. All three study areas have $6 \times 6 \mathrm{~km}$ area. Pi-SAR2 images were projected to the WGS 1984 coordinate system with UTM (Universal Transverse Mercator) projection. The Quegan's polarimetric calibration procedure (Quegan, 1994) was used for polarimetric calibration.

\begin{tabular}{|c|c|}
\hline Center frequency & $9.55 / 9.65 \mathrm{GHz}$ \\
Bandwidth & $500 / 300 / 150 \mathrm{MHz}$ \\
Slant-range resolution & $0.3 / 0.5 / 1.0 \mathrm{~m}$ \\
Azimuth resolution & $0.3(1$ look $) / 0.6 \mathrm{~m}(2$ look $)$ \\
Swath width (grand range) & $>10 \mathrm{~km}$ \\
NESZ & $<-23 /-27 /-30 \mathrm{~dB}$ \\
Data rate & $200 \mathrm{MB} / \mathrm{s} \times 4 \mathrm{ch}$ \\
\hline
\end{tabular}

Table 1. Specification of Pi-SAR2

\section{METHOD AND RESULTS}

\subsection{Outline}

Figure 2 shows the flowchart of the proposed method. First, as preprocessing, multilook processing is implemented and four components are generated with correction of the polarization orientation angle (POA) effect. Next, the first classification using total power of scattering $(T P)$ and $P_{v}$ is implemented. Then, the second classification using polarimetric coherency is implemented. After both results are intersected, finally, urban areas are extracted.

\subsection{Four Component Decomposition and Multilooing}

The format of PolSAR data consists of a complex scattering matrix expressed by Equation (1):

$$
s=\left(\begin{array}{cc}
S_{H H} & S_{H V} \\
S_{V H} & S_{V V}
\end{array}\right)=\left(\begin{array}{cc}
a & c \\
c & b
\end{array}\right)
$$

Here, for simplicity, $S_{H V}$ and $S_{V H}$ are assumed to be equivalent. From a scattering matrix, the coherency matrix is derived by Equation (2):

$$
\begin{aligned}
& T=\left(\begin{array}{lll}
T_{11} & T_{12} & T_{13} \\
T_{21} & T_{22} & T_{23} \\
T_{31} & T_{32} & T_{33}
\end{array}\right) \\
& =\frac{1}{2}\left(\begin{array}{ccc}
|a+b|^{2} & (a+b)(a-b)^{*} & 2(a+b) c^{*} \\
(a-b)(a+b)^{*} & |a-b|^{2} & 2(a-b) c^{*} \\
2 c(a+b)^{*} & 2 c(a-b)^{*} & 4|c|^{2}
\end{array}\right)
\end{aligned}
$$

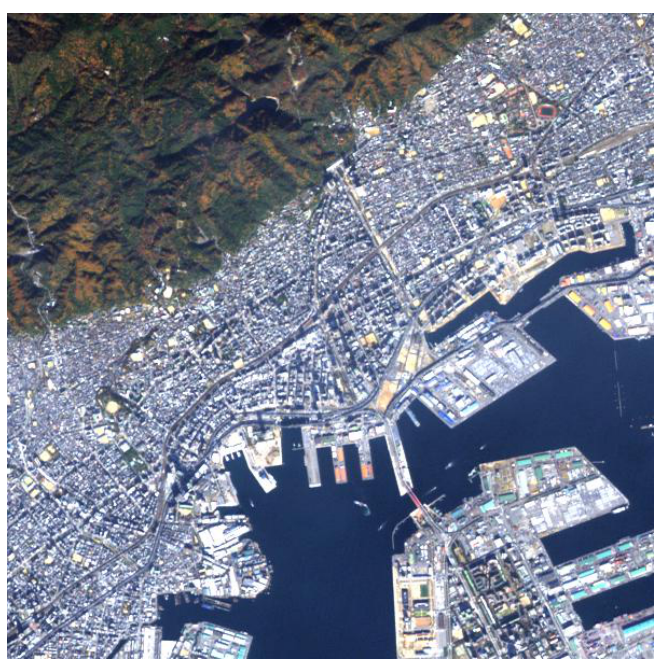

(a)

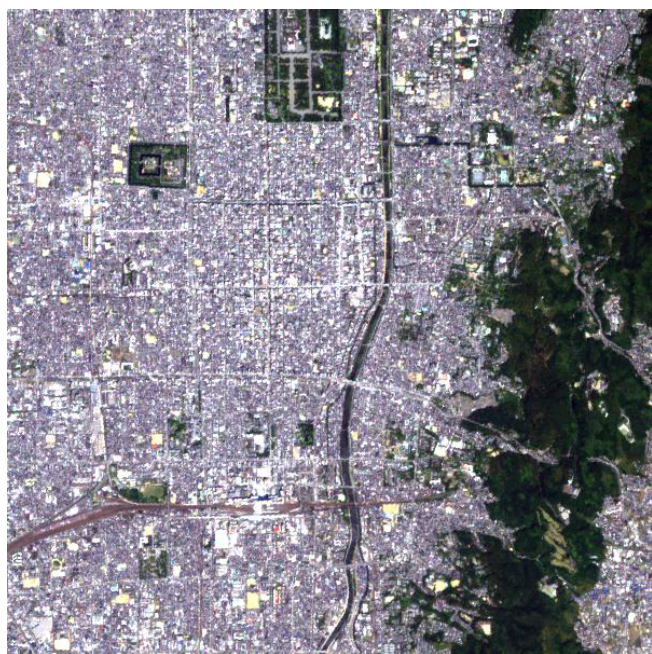

(b)

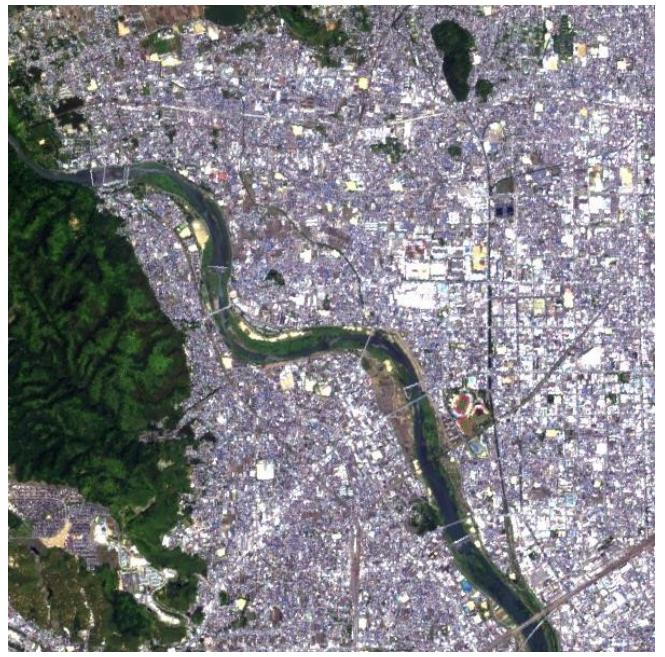

(c)

Figure 1. ALOS/AVNIR-2 images of (a) study area 1, (b) study area 2 and (c) study area 3 (R:G:B=Band3:2:1). The image of study area 1 was acquired on December 2, 2008 and the image of study areas 2 and 3 was acquired on May 18, 2009. 


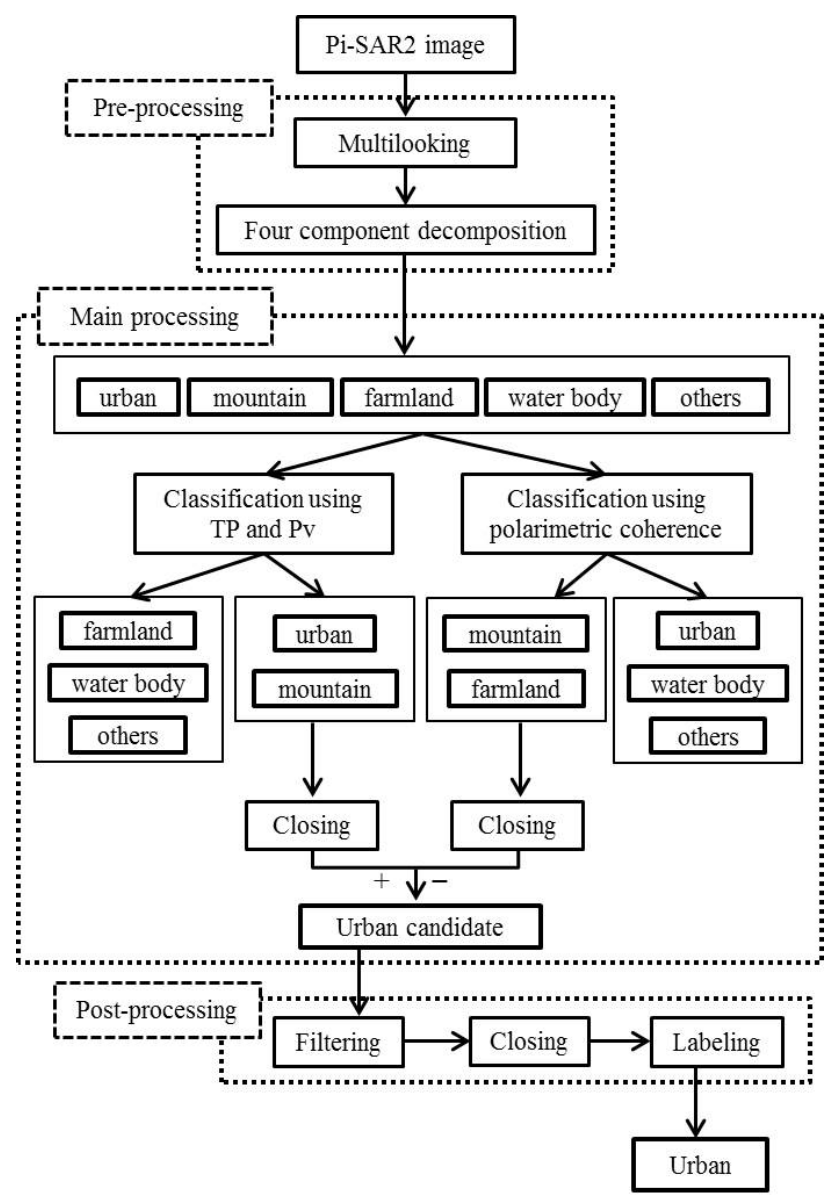

Figure 2. Flowchart of extracting urban areas using Pi-SAR2 images.

From the coherency matrix, four-component decomposition decomposes the observed backscattering into four components, the surface scattering power $\left(P_{s}\right)$, the double-bounce scattering power $\left(P_{d}\right)$, the volume scattering power $\left(P_{v}\right)$, and the helix scattering power $\left(P_{c}\right)$ (Yamaguchi et al., 2005). Yamaguchi et al. (2006) proposed an algorithm that rotates the coherency matrix by the POA in order to reduce the dependence of the components on the relative azimuth. The POA is defined by Equation (3).

$$
\theta=\frac{1}{4} \tan ^{-1} \frac{2 \operatorname{Re}\left(T_{23}\right)}{T_{22}-T_{33}}
$$

A rotation is applied to the coherency matrix by using the POA:

$$
\begin{aligned}
T(\theta) & =\left(\begin{array}{lll}
T_{11}(\theta) & T_{12}(\theta) & T_{13}(\theta) \\
T_{21}(\theta) & T_{22}(\theta) & T_{23}(\theta) \\
T_{31}(\theta) & T_{32}(\theta) & T_{33}(\theta)
\end{array}\right) \\
& =R_{p}(\theta) T R_{p}(\theta)^{\dagger} .
\end{aligned}
$$

Here, $\uparrow$ denotes complex conjugation and transposition, and $R_{p}(\theta)$ is the rotation matrix given by

$$
R_{p}(\theta)=\left(\begin{array}{ccc}
1 & 0 & 0 \\
0 & \cos 2 \theta & \sin 2 \theta \\
0 & -\sin 2 \theta & \cos 2 \theta
\end{array}\right)
$$

The coherency matrix is decomposed into four components:

$$
\langle T\rangle=f_{s}\left\langle T_{s}\right\rangle+f_{d}\left\langle T_{d}\right\rangle+f_{v}\left\langle T_{v}\right\rangle+f_{c}\left\langle T_{c}\right\rangle
$$

Here, $f_{s}, f_{d}, f_{v}$ and $f_{c}$ denote coefficients of the surface, doublebounce, volume and helix scatterings, respectively, and $T_{s}, T_{d}, T_{v}$ and $T_{c}$ denote coherency matrices of the surface, double-bounce, volume and helix scatterings, respectively. Four component decomposition method calculates the four coefficients and thus the contribution of each scattering is determined.

Prior to the four component decomposition, multilooking is conducted. While we used VHR SAR images, we focused on the extraction of urban areas, that does not require a high spatial resolution product, e.g. sub-meter level. Considering the computation time for calculating $6 \times 6 \mathrm{~km}$ area, we determined pixel spacing as $2.5 \mathrm{~m}$.

\subsection{Classification Using $T P$ and $P_{v}$}

Following a part of the method proposed by Kajimoto and Susaki (2013a), we use $T P$ and $P_{v}$ for the first classification. Because of dependency on POA, the method proposed by Kajimoto and Susaki (2013a) set different thresholds based on POA space. However, because the dependency of Pi-SAR2 data on POA was not significant, we applied the method without dividing POA space into sub-spaces.

First, urban areas and mountains training data are manually selected from study area 2 because the aspects of mountains in study area 2 are more random than other study areas. Next, principal component analysis is applied to the training data, and the threshold for discriminating urban areas from mountains is determined. The threshold was determined from the means and standard deviations of the first principal components of the two land cover types. The difference between the means of the two land covers was divided in proportion to the standard deviations, and the break point was obtained. Thereafter, the second component was shifted along the first component as passing the break point, and the shifted second component was used as the threshold. The threshold is then applied to study areas 1 and 3, and an attempt is made to separate the urban areas from mountains. The difference is calculated between the urban gravity points in the new area and in the initial study area, and this difference is used to adjust the threshold. The updated threshold is again applied to the new study area, and the gravity point difference is calculated. Iteration of this process is terminated when the change in the threshold is within a predefined limit. As a results, three lines for discriminating urban areas from mountains, expressed by Equation (7), were obtained.

$$
\begin{array}{ll}
\text { study area 1: } & T P=-0.902 P_{v}-69.89 \\
\text { study area 2: } & T P=-0.902 P_{v}-42.10 \\
\text { study area 3: } & T P=-0.902 P_{v}-54.68
\end{array}
$$

Units of $\mathrm{TP}$ and $\mathrm{Pv}$ are $\mathrm{dB}$, respectively. The result generated at this stage, shown in Figure 3, includes urban areas and a part of mountains. The remaining mountains correspond to the ridges that return strong backscatters. In addition, the extracted urban areas mainly correspond to buildings that are isolated. To generate an urban area map, not building distribution map, we applied closing processing (dilation and erosion) twice.

\subsection{Classification Using Polarimetric Coherence}

Kajimoto and Susaki (2013a) proposed a method to discriminate urban areas from mountains by using spatial randomness of POA, and demonstrated that the method was effective to L-band PALSAR images. However, we found that such approach was not effective to X-band Pi-SAR2 images. It may be partly because of radar frequency difference and spatial resolution difference. After we examined other effective approaches, we determined 


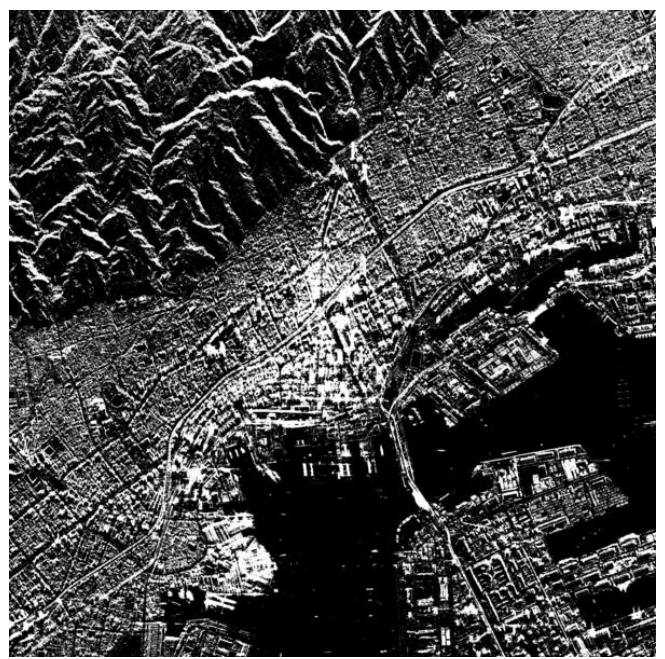

Figure 3. Classification result using $T P$ and $P_{v}$ before closing in study area 1

that polarimetric coherence between $S_{H H}$ and $S_{V V}$, expressed by Equation (8) is a good indicator for the purpose.

$$
\gamma_{H H, V V}=\frac{\left|\left\langle S_{H H} S_{V V}^{*}\right\rangle\right|}{\sqrt{\left\langle S_{H H} S_{H H}^{*}\right\rangle\left\langle S_{V V} S_{V V}^{*}\right\rangle}}
$$

Polarimetric coherence is sensitive to surface condition. Because urban areas have various artifacts, they may show lower values than mountains and farmland. In the experiment, the ensemble average was calculated within a $5 \times 5$ window, and the threshold for $\gamma_{H H, V V}$ was set to 0.9 from an empirical examination. Figure 4 shows that the higher polarimetric coherence correspond to mountains and farmland. In the same way as classification using $T P$ and $P_{v}$, we applied closing to the result two times.

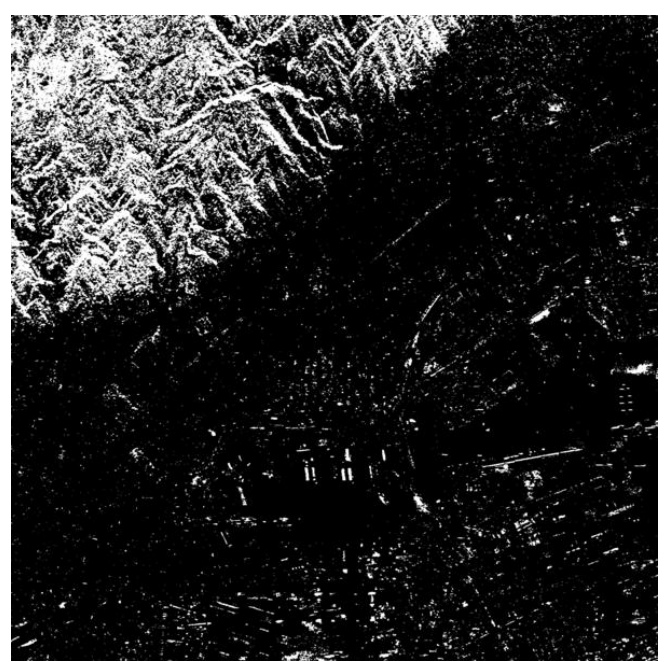

Figure 4. Classification result using $\gamma_{H H, V V}$ before closing in study area 1

\subsection{Intersection and Post-classification}

Figures 3 and 4 indicate that the mountains extracted by using $\gamma_{H H, V V}$ include the mountains remaining in the result using $T P$ and $P_{v}$. Therefore, we intersected the image obtained by using $T P$ and $P_{v}$ and the inverse image obtained by using $\gamma_{H H, V V}$.
The result is shown in Figure 5(a). This intersected image, named as "urban candidates", shows urban areas are well extracted, but roads and radar shadow are not extracted. Therefore, we applied filtering, closing (twice) and labeling to the urban candidate image. Filtering examined the percentage of urban candidate pixels within a window. We set the window size to $5 \times 5$, and the minimum percentage for urban areas to $20 \%$. Labeling is implemented to remove small areas. The minimum urban area was set to 500 pixels, equivalent to $3,125 \mathrm{~m}^{2}$. Figures $5(\mathrm{~b})$ and 5 (c) show the result generated by filtering and closing and the final urban areas, respectively.

Figure 6 shows the classification results using $T P$ and $P_{v}$, those using $\gamma_{H H, V V}$, and final urban areas in study area 2 . Figure 7 show the results in study area 3 .

\subsection{Accuracy Assessment}

Urban area data were obtained from Zmap-TOWN II (ZENRIN) data, which are residential maps of Japan (Zenrin, 2014). First, building polygon data were intersected by a mesh. Buildings lying across the mesh border are divided into pieces by the border line. The mesh size was set to $100 \mathrm{~m} \times 100 \mathrm{~m}$. Then, the mesh was classified into urban category or non-urban category. The minimum percentage of buildings within a mesh for classifying into urban category was set to $20 \%$. As a result, a reference map of urban area was generated. Finally, the urban areas extracted from Pi-SAR2 images were compared with the reference map.

As a reference method, we focused on Esch et al. (2013). They extracted urban areas by using backscatter amplitude and speckle divergence obtained from single-polarization TerraSAR-X and TanDEM-X data. Figures 8 and 9 show the extracted urban areas of study areas 1 and 2, respectively. Figures 8(a) and 9(a), and $8(\mathrm{c})$ and $9(\mathrm{c})$ show the extracted urban areas using the proposed method and the method of Esch et al.(2013) without postprocessing, respectively. Similary, Figures 8(b) and 9(b), and 8 (d) and 9(d) show the extracted urban areas using the proposed method and the method of Esch et al.(2013) with post-processing, respectively. Tables 2 and 3 show the accuracy assessment results of study area 1 by using the method of Esch(2013) and the proposed method, respectively. Similarly, Tables 4 and 5 show the accuracy assessment results of study area 1 by using the method of Esch(2013) and the proposed method, respectively.

\section{DISCUSSION}

\subsection{Comparison with Existing Method}

Tables 2 to 5 show the accuracies of the proposed method are higher than those of the method of Esch et al.(2013). As shown in Figures 8(b) and 8(d), in study area 1, the method proposed by Esch et al. (2013) cannot remove mountains in layovered areas compared to the proposed method. Because of off nadir angle difference, airborne SAR often faces layover compared with spaceborne SAR. In study area 2, it was found that the hilly urban areas were missed by the method of Esch et al.(2013) whereas the proposed method extracted them. Therefore, the proposed method is more effective to extract urban areas by using airborne SAR data than the method of Esch et al.(2013).

\subsection{Post-classification}

In terms of mapping urban areas, classification using VHR Xband SAR images needs several processes. Figures 5(a), 6(a) and 7 (a) show clear contrast between buildings and roads. They indicate that VHR X-band images can extract buildings, but that 


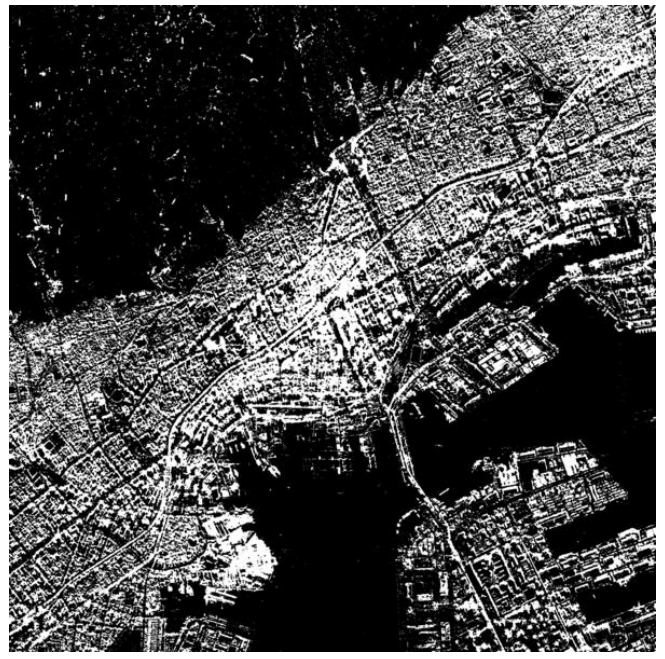

(a)

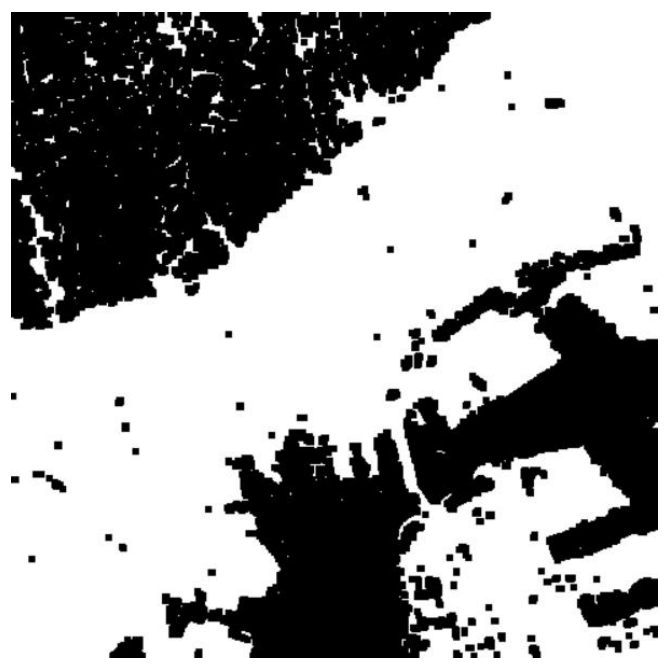

(b)

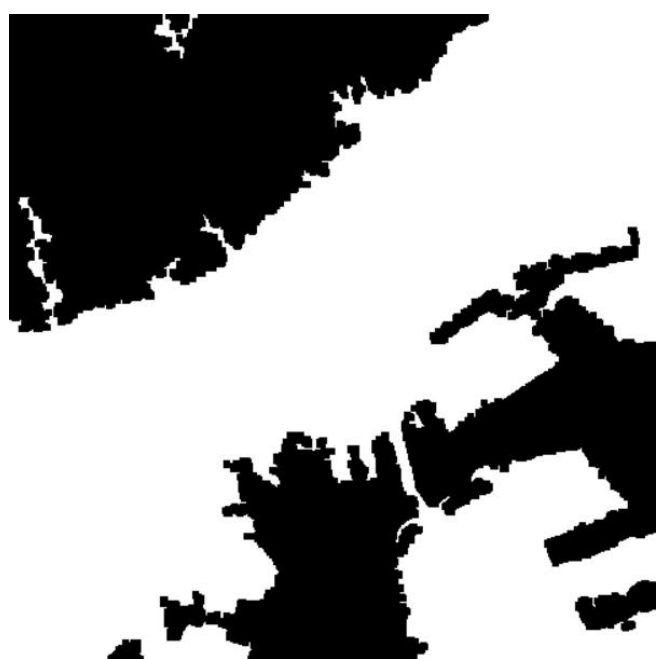

(c)

Figure 5. Urban areas in study area 1. (a) Urban candidate areas generated by intersection of Figures 3(b) and 4(b), (b) result generated by filtering and closing (a), and (c) final result generated by labeling (b).

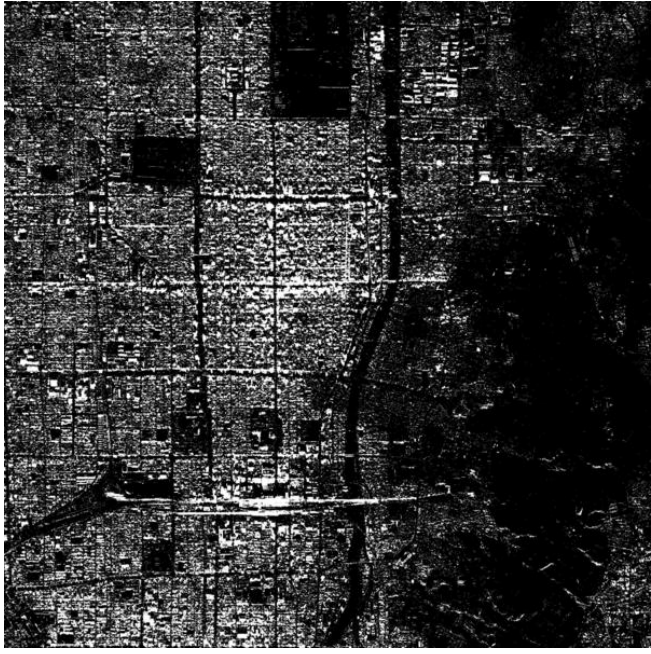

(a)

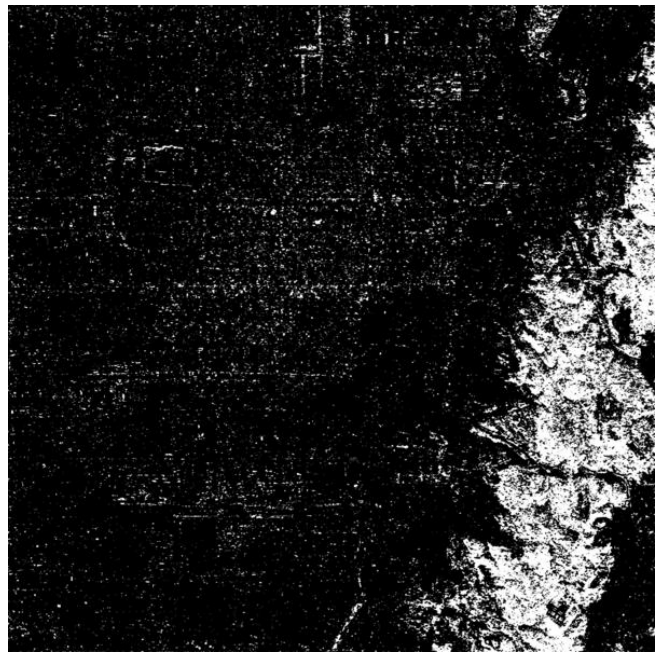

(b)

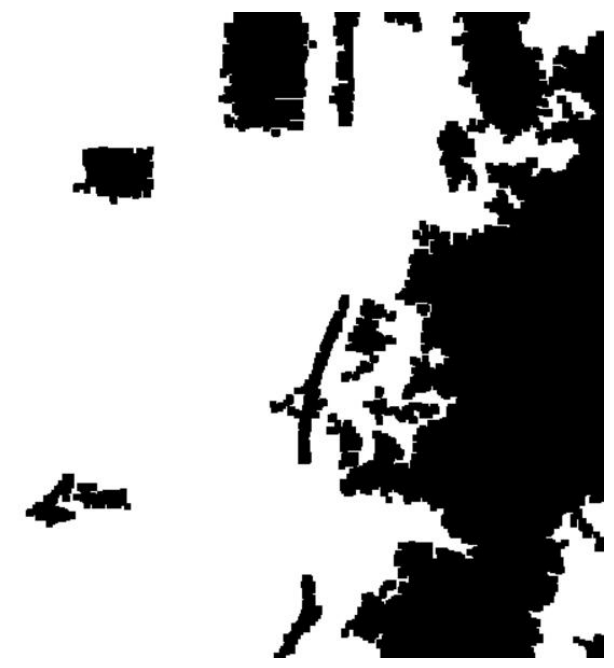

(c)

Figure 6. Urban areas in study area 2. (a) Classification result using $T P$ and $P_{v}$ without closing, (b) classification result using $\gamma_{H H, V V}$ without closing, and (c) final result. 


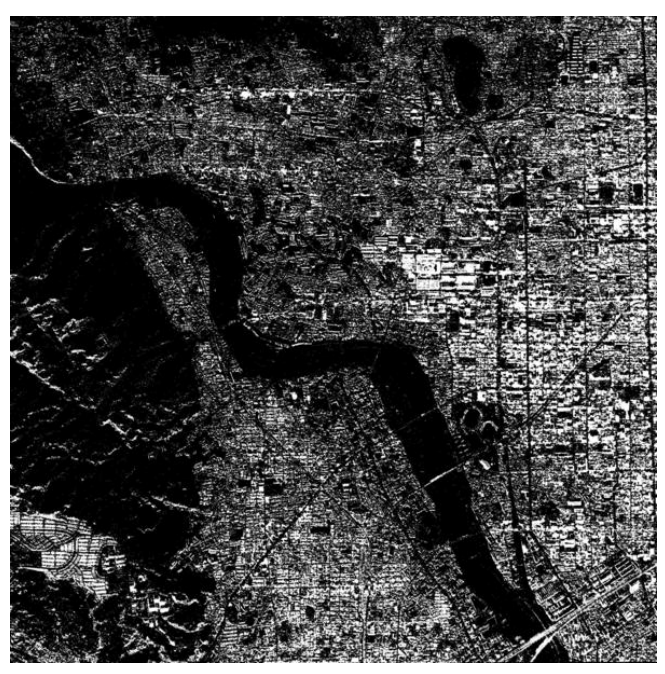

(a)

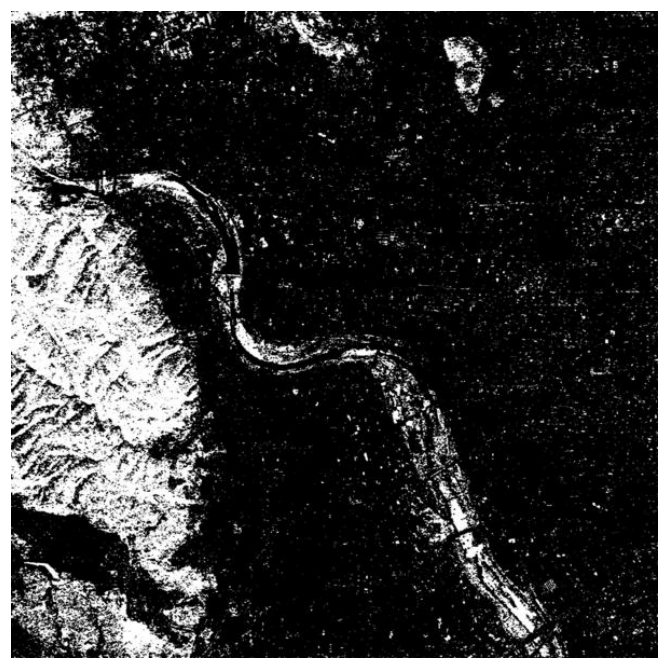

(b)

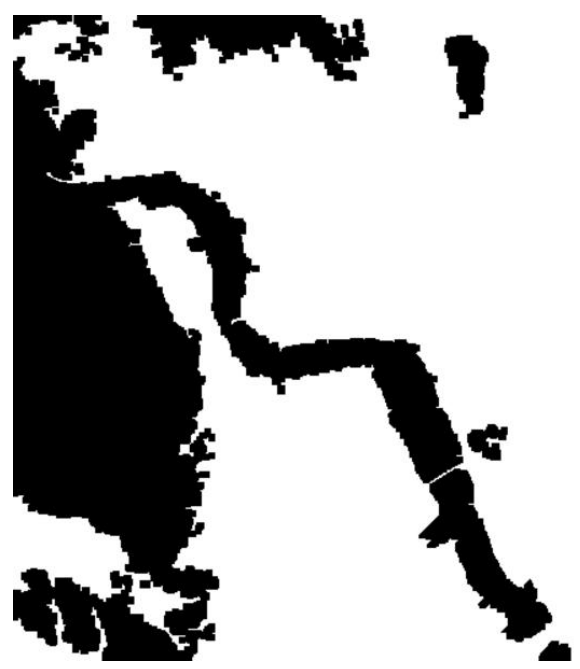

(c)

Figure 7. Urban areas in study area 3. (a) Classification result using $T P$ and $P_{v}$ without closing, (b) classification result using $\gamma_{H H, V V}$ without closing, and (c) final result.

\begin{tabular}{|c|c|rrr|r|}
\hline \multicolumn{2}{|c|}{$\begin{array}{c}\text { Kobe } \\
\text { Esch(2013) }\end{array}$} & \multicolumn{3}{|c|}{ Reference } & Producer's \\
\cline { 2 - 4 } Est. & Urban & Others & Total & accuracy \\
\hline \multirow{3}{*}{ Est. } & Urban & 1519 & 842 & 2361 & $64.3 \%$ \\
& Others & 10 & 1229 & 1239 & $99.2 \%$ \\
& Total & 1529 & 2071 & 3600 & \\
\hline \multicolumn{2}{|c|}{ User's accuracy } & $99.3 \%$ & $59.3 \%$ & \\
\hline \multicolumn{5}{|c|}{ Overall accuracy } & $76.3 \%$ \\
\hline
\end{tabular}

Table 2. Accuracy of extracted urban areas in study area 1 by using the method of Esch et al.(2013)

\begin{tabular}{|c|c|rrr|r|}
\hline \multicolumn{2}{|c|}{ Kobe } & \multicolumn{3}{|c|}{ Reference } & Producer's \\
Proposed method & Urban & Others & Total & accuracy \\
\hline \multirow{3}{*}{ Est. } & Urban & 1522 & 762 & 2284 & $66.6 \%$ \\
& Others & 7 & 1309 & 1316 & $99.5 \%$ \\
& Total & 1529 & 2071 & 3600 & \\
\hline \multicolumn{2}{|c|}{ User's accuracy } & $99.5 \%$ & $63.2 \%$ & \\
\hline \multicolumn{4}{|c|}{ Overall accuracy } & $78.5 \%$ \\
\hline
\end{tabular}

Table 3. Accuracy of extracted urban areas in study area 1

\begin{tabular}{|c|c|c|c|c|c|}
\hline \multirow{2}{*}{\multicolumn{2}{|c|}{$\begin{array}{c}\text { Kyoto } \\
\text { Esch(2013) }\end{array}$}} & \multicolumn{3}{|c|}{ Reference } & \multirow{2}{*}{$\begin{array}{r}\text { Producer's } \\
\text { accuracy }\end{array}$} \\
\hline & & \multirow{2}{*}{$\begin{array}{r}\text { Urban } \\
2160\end{array}$} & \multirow{2}{*}{$\begin{array}{r}\text { Others } \\
142\end{array}$} & \multirow{2}{*}{$\begin{array}{l}\text { Total } \\
2302\end{array}$} & \\
\hline \multirow{3}{*}{ Est. } & Urban & & & & $93.8 \%$ \\
\hline & Others & 510 & 788 & 1298 & \multirow{2}{*}{$60.7 \%$} \\
\hline & Total & 2670 & 930 & 3600 & \\
\hline \multicolumn{2}{|c|}{ User's accuracy } & $80.9 \%$ & \multicolumn{2}{|c|}{$54.8 \%$} & \\
\hline & & \multicolumn{3}{|c|}{ Overall accuracy } & $81.9 \%$ \\
\hline
\end{tabular}

Table 4. Accuracy of extracted urban areas in study area 2 by using the method of Esch et al.(2013)

\begin{tabular}{|c|c|rrr|r|}
\hline \multicolumn{2}{|c|}{ Kyoto } & \multicolumn{3}{|c|}{ Reference } & Producer's \\
\cline { 2 - 4 } Proposed method & Urban & Others & Total & accuracy \\
\hline \multirow{3}{*}{ Est. } & Urban & 2431 & 214 & 2645 & $91.9 \%$ \\
& Others & 239 & 716 & 955 & $75.0 \%$ \\
& Total & 2670 & 930 & 3600 & \\
\hline \multicolumn{2}{|c|}{ User's accuracy } & $91.0 \%$ & $77.0 \%$ & \\
\hline \multicolumn{4}{|c|}{ Overall accuracy } & $87.4 \%$ \\
\hline
\end{tabular}

Table 5. Accuracy of extracted urban areas in study area 2 
mapping of urban areas should include other objects in urban areas. To deal with this issue, we took an approach that the results of classification should be extended by applying closing filtering. Such approaches can compensate the areas where radar shadow occurs. We suggest that extraction of urban areas using VHR Xband SAR images should be accompanied by such algorithms to extract widely homogeneous areas.

\section{CONCLUSIONS}

In this paper, we presented a method to extract urban areas from $\mathrm{X}$-band PolSAR data. The proposed method is based on two classifications: classification with combination of $T P$ and $P_{v}$, and classification using polarimetric coherency between $S_{H H}$ and $S_{V V}$. The two results are intersected and final urban areas are extracted after post-classification processing. We applied the proposed method to airborne X-band Pi-SAR2 images of Kobe and Kyoto, Japan. The validation show that the results of the proposed method were acceptable, with an overall accuracy of approximately 80 to $90 \%$ at 100 -m spatial scale. It was also shown that extraction of mountains using X-band PolSAR images is more stable than that using L-band PolSAR images. Whereas the sensitivity analysis revealed that the most sensitive threshold for extracting urban areas was that for $\gamma_{H H, V V}$, it was confirmed that the extracted results were stable in most cases. We conclude that the proposed method can be applied to X-band PolSAR images for robustly extracting urban areas.

\section{ACKNOWLEDGEMENTS}

This research was supported in part by a program of the PiSAR-2 research announcement of NICT, Japan.

\section{REFERENCES}

Brunner, D., Lemoine, G., Bruzzone, L. and Greidanus, H., 2010. Building height retrieval from VHR SAR imagery based on an iterative simulation and matching technique, IEEE Trans. Geosci. Remote Sens., 48, pp. 1487 - 1504.

Brunner, D., Lemoine, G., Greidanus, H. and Bruzzone, L., 2011. Radar imaging simulation for urban structures, IEEE Geosci. Remote Sens. Letters, 8, pp. 68 - 72.

Cossu, R., Dell'Acqua, F., Polli, D.A. and Rogolino, G., 2012. SAR-based seismic damage assessment in urban areas: Scaling down resolution, scaling up computational performance, IEEE J. Selected Topics App. Earth Observ. Remote Sens., 5, pp. 1110 1117.

Esch, T., Marconcini, M., Felbier, A., Roth, A., Heldens, W., Huber, M., Schwinger, M., Taubenbock, H., Muller, A. and Dech, S., 2013. Urban footprint processor-fully automated processing chain generating settlement masks from global fata of the TanDEM-X mission, IEEE Trans. Geosci. Remote Sens. Letters, 10, pp. $1617-1621$.

Ferro, A., Brunner, D., Bruzzone, L. and Lemoine, G., 2011. On the relationship between double bounce and the orientation of buildings in VHR SAR images, IEEE Geosci. Remote Sens. Letters, 8, pp. 612 - 616.

Ferro, A., Brunner, D. and Bruzzone, L., 2013. Automatic detection and reconstruction of building radar footprints from single VHR SAR images, IEEE Trans. Geosci. Remote Sens., 51, pp. 935 - 952.
Gamba, P., Aldrighi, M. and Stasolla, M., 2011, Robust extraction of urban area extents in HR and VHR SAR images, IEEE J. Selected Topics App. Earth Observ. Remote Sens., 4, pp. 27 - 34.

Kajimoto, M. and Susaki, J., 2013a. Urban-Area Extraction From Polarimetric SAR Images Using Polarization Orientation Angle, IEEE Geosci. Remote Sens. Letters, 10, pp. 337 - 341.

Kajimoto, M. and Susaki, J., 2013b. Urban density estimation from polarimetric SAR images based on a POA correction method, IEEE J. Selected Topics App. Earth Observ. Remote Sens., 6, pp. $1418-1429$.

Kimura, H., 2008. Radar polarization orientation shifts in builtup areas. IEEE Geosci. Remote Sens. Letters, 5, 217-221.

Kobayashi, T., Umehara, T., Uemoto, J., Satake, M., Kojima, S., Matsuoka, T., Nadai, A. and Uratsuka, S., 2013. Volcanic monitoring by polarimetric and interferometric airborne SAR (PiSAR2), Proceedings of APSAR 2013, pp. 487 - 490.

Marin, C., Bovolo, F. and Bruzzone, L., 2014. Building change detection in multitemporal very high resolution SAR images, IEEE Trans. Geosci. Remote Sens., 53, pp. 2664 - 2682.

Quegan, S., 1994. A unified algorithm for phase and cross-talk calibration of polarimetric data-theory and observations, IEEE Trans. Geosci. Remote Sens., 32, pp. 89 - 99.

Satake, M., Kobayashi, T., Uemoto, J., Umehara, T., Kojima, S., Matsuoka, T., Nadai, A. and Uratsuka, S., 2012. Damage estimation of the Great East Japan earthquake with airborne SAR (PI-SAR2) data, Proceedings of IGARSS 2012, pp. 1190 - 1191.

Susaki, J., Kajimoto, M. and Kishimoto, M. 2014. Urban density mapping of global megacities from polarimetric SAR images, $R e$ mote Sens. Environ., 155, pp. 334 - 348.

Uemoto, J., Uratsuka, S., Umehara, T., Yamamoto, S.-I., Taira, S., Satake, M., Kojima, S., Kobayashi, T., Satoh, M., Kawasaki, K., Matsuoka, T., Nadai, A. and Suzuki, R., 2011. Development of the onboard processor for Pi-SAR2, Proceedings of IGARSS 2011, pp. 906 - 909.

Yamaguchi, Y., Moriyama, T., Ishido, M. and Yamada, H., 2005. Four-component scattering model for polarimetric SAR image decomposition, Geoscience and Remote Sensing, IEEE Trans. Geosci. Remote Sens., 43, pp. 1699 - 1706.

Yamaguchi, Y., Yajima, Y. and Yamada, H., 2006. A four-component decomposition of POLSAR images based on the coherency matrix, IEEE Geosci. Remote Sens. Letters, 3, pp. 292 - 296.

Zenrin, Co. Ltd, 2014. Zmap Town II. Available at http://www. zenrin.co.jp/product/gis/zmap/zmaptown.html (in Japanese: accessed Nov 20, 2014) 


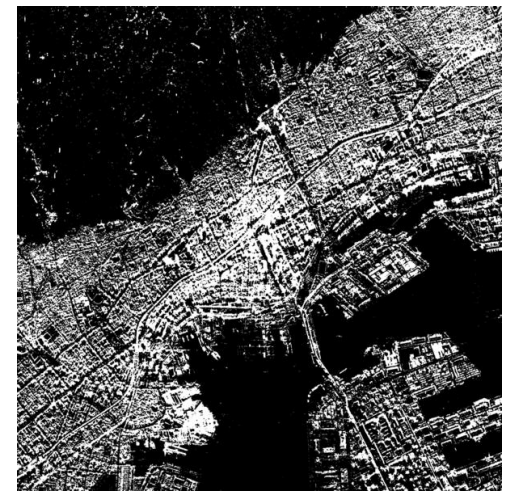

(a)

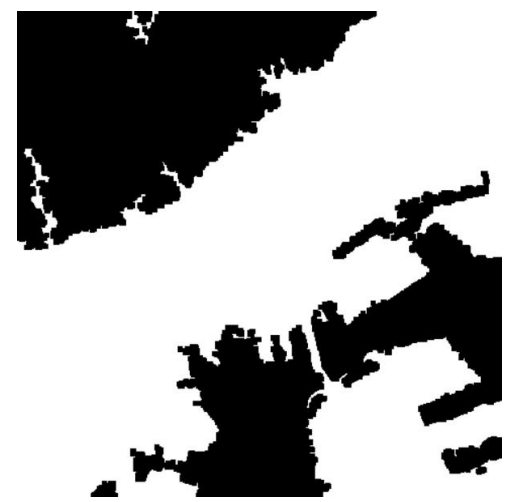

(b)

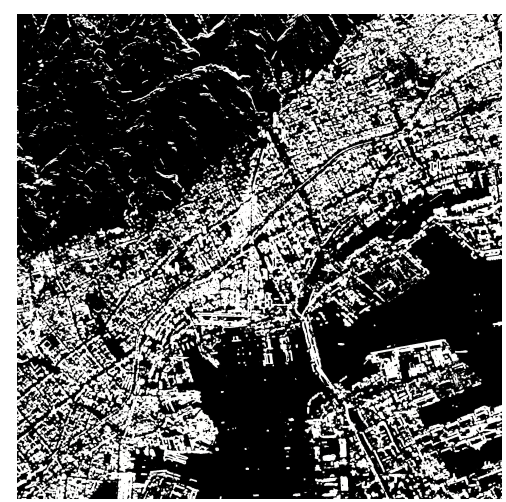

(c)

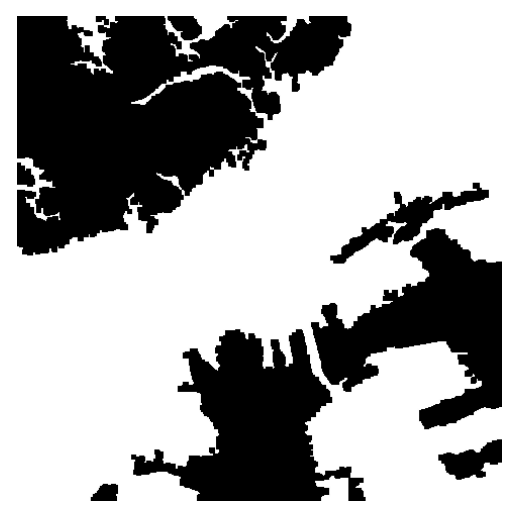

(d)

Figure 8. Comparison of classification results in study area 1 . (a)(b) Results by using the proposed method before and after post-processing, respectively, and (c)(d) the result by using the method of Esch et al.(2013) before and after post-processing, respectively.

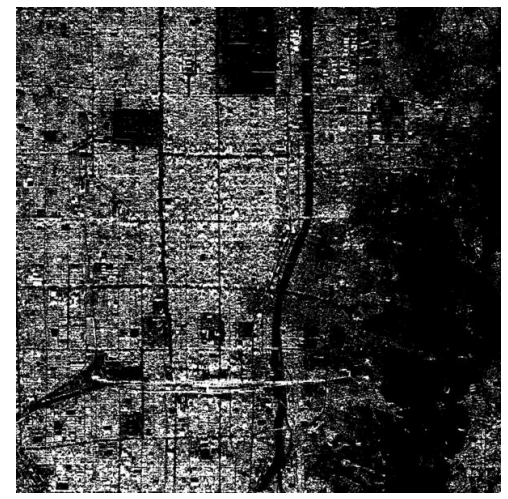

(a)

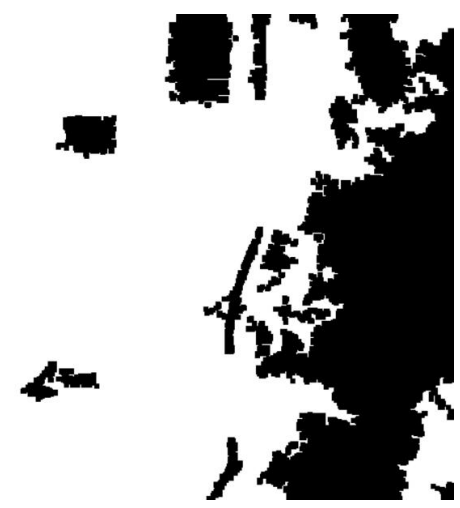

(b)

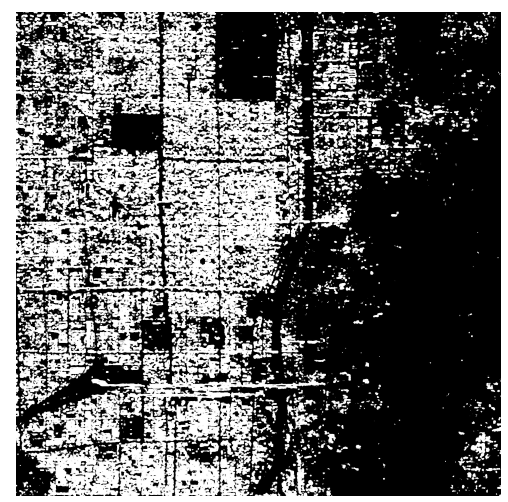

(c)

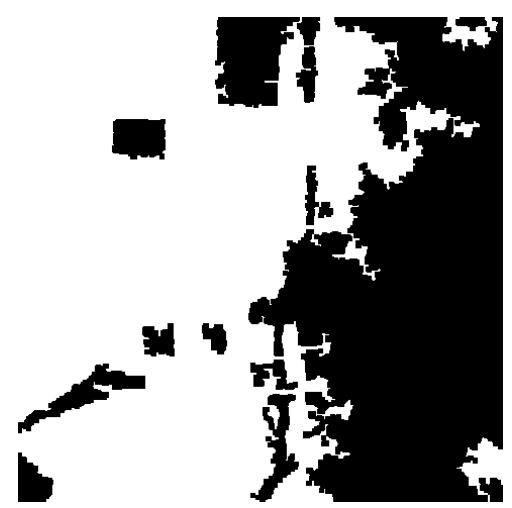

(d)

Figure 9. Comparison of classification results in study area 2. (a)(b) Results by using the proposed method before and after post-processing, respectively, and (c)(d) the result by using the method of Esch et al.(2013) before and after post-processing, respectively. 